\section{Sub-1 V CMOS large capacitive-load driver circuit using direct bootstrap technique for low-voltage CMOS VLSI}

\author{
P.C. Chen and J.B. Kuo
}

A novel sub-1 V CMOS large capacitive-load driver circuit using a direct bootstrap technique for low-voltage CMOS VLSI is reported. For a supply voltage of $1 \mathrm{~V}$, the CMOS large capacitive-load driver circuit using the direct bootstrap technique shows a 3.3 times improvement in switching speed in driving a capacitive load of $2 \mathrm{pF}$ compared to the conventional bootstrapped CMOS driver circuit using an indirect bootstrap technique. Even for a supply voltage of $0.8 \mathrm{~V}$, this CMOS large capacitive load driver circuit using the direct bootstrap technique is still advantageous.

Introduction: Nowadays the design of the driver circuit is the key step in determining the speed performance of a CMOS VLSI circuit with a large capacitive load. Indirect bootstrap techniques to raise/lower the gate voltage of the output NMOS/PMOs device have been used in the large load driver circuit using a low power supply voltage [1-3]. Since the bootstrap technique is applied at the gate of the output devices in a driver circuit, it is categorised as the indirect bootstrap technique. The indirect bootstrap technique via the gate of the output device may not be effective in shortening the switching speed of the output. Recently, low power supply voltage is the trend on the future CMOS VLSI circuits. Since the magnitude of the threshold voltage of the CMOS devices cannot be scaled down accordingly with the power supply voltage, the design of an effective large load driver circuit using down-scaled CMOS devices with a low power supply voltage is challenging. In this letter we describe an effective large load CMOS driver circuit using a novel direct bootstrap technique applied on the output. The direct bootstrap technique used in a large load CMOS driver circuit is described first, followed by the performance evaluation and discussion.

Direct bootstrap technique: Fig. 1 shows the sub-1 V large load driver circuit with the direct bootstrap technique. As shown in the Figure, this circuit is composed of three NMOS devices N1-N3, three PMOS devices P1-P3, and two inverters. In addition, two bootstrap capacitors $C_{B 1}$ and $C_{B 2}$ have been used to implement the direct bootstrap technique. Among these devices, PMOS devices P1-P3 and the bootstrap capacitor $C_{B 1}$ are used for the pullup operation; NMOS devices N1-N3 and the bootstrap capacitor $C_{B 2}$ for the pulldown operation. In this direct bootstrap technique, the bootstrap capacitor $\mathrm{C}_{\mathrm{B} 1} / \mathrm{C}_{\mathrm{B} 2}$ is connected to the output node via $\mathrm{P} 2 / \mathrm{N} 2$, instead of via the gate of the output device as in other indirect bootstrap techniques [1-3]

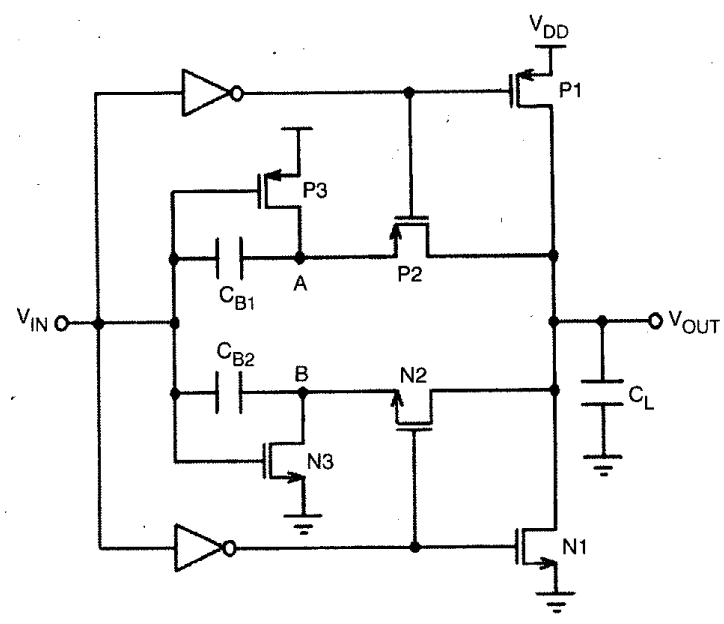

Fig. 1 Sub-I V CMOS large capactive load driver circuit using direct bootstrap technique

Performance and discussion: To assess the performance of the large load driver circuit using the direct bootstrap technique, a test circuit based on a $0.18 \mu \mathrm{m}$ CMOS technology has been designed. The channel length of all devices in the circuit is $0.18 \mu \mathrm{m}$. The channel width of the NMOS/PMOS devices is $40 \mu \mathrm{m} / 80 \mu \mathrm{m}$. Fig. 2 shows the transient waveforms of the sub-1V large load CMOS driver circuit using the direct bootstrap technique with an output load of $1 \mathrm{pF}$ and the bootstrap capacitors of $0.35 \mathrm{pF}$ at a power supply voltage of $1 \mathrm{~V}$ based on SPICE simulation results. As shown in Fig. $3 a$, prior to the pullup operation, the input voltage $\mathrm{V}_{\mathrm{in}}=0 \mathrm{~V}$. P3 is on and $\mathrm{N} 2$ is on. Under this situation, the bootstrap capacitor $\mathrm{C}_{\mathrm{B} 1}$ is charged to $1 \mathrm{~V}$ since its right side is connected to $1 \mathrm{~V}$ via $\mathrm{P} 3$ and the left side is at $0 \mathrm{~V}$. At the same time, the bootstrap capacitor $C_{B 2}$ is charged to $0 \mathrm{~V}$ since both sides are connected to $0 \mathrm{~V}$. As shown in Fig. $3 b$, after the input ramps up to $1 \mathrm{~V}, \mathrm{~N} 3$ is on and $\mathrm{P} 2$ is on. Therefore, the right side of the bootstrap capacitor $\mathrm{C}_{\mathrm{B} 1}$ is connected to the output node. Owing to the charge stored in the bootstrap capacitor $C_{B 1}$, from the positive jump at the input from 0 to $1 \mathrm{~V}$, the internal node $\mathrm{A}$ is bootstrapped to $1.35 \mathrm{~V}$. As a result, the output $\mathrm{V}_{\text {out }}$ is quickly raised to $1 \mathrm{~V}$. As shown in the Figure, the output $V_{\text {out }}$ may surpass 1 to $1.1 \mathrm{~V}$ for a short period time. During the pulldown operation, the scheme is complementary. Prior to the pulldown operation, the bootstrap capacitor $C_{B 2}$ is charged to $-1 \mathrm{~V}$ via $\mathrm{N} 3$. After the input ramp-down period, owing to the stored charge in the bootstrap capacitor $\mathrm{C}_{\mathrm{B} 2}$, the internal node $\mathrm{B}$ is bootstrapped to below $0 \mathrm{~V}$. As a result, the pulldown time of the output has been shortened.

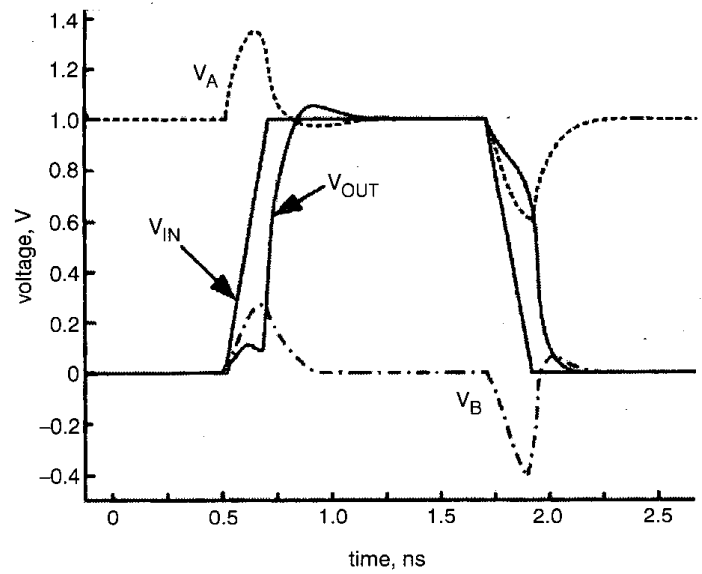

Fig. 2 Transient waveforms during pullup operation of sub-1 V CMOS large capactive load driver circuit with direct bootstrap technique Bootstrap capacitors $0.35 \mathrm{pF}$, output load $2 \mathrm{pF}$, at power supply voltage of $1 \mathrm{~V}$

Fig. 4 shows the pullup propagation delay time against load capacitance of the large load CMOS driver circuit using the direct bootstrap technique with bootstrap capacitors of 1 and $0.35 \mathrm{pF}$. Also shown in the Figure are the results of the driver circuits using the indirect bootstrap techniques [1-3]. For a load capacitance of 2 pf, the speed improvement of the driver circuit using the direct bootstrap technique over the indirect bootstrap one [1] is 3.3 times. Different from the other indirect bootstrap one, the direct bootstrap technique used in this driver circuit is more effective in enhancing the switching speed. The effectiveness of the direct bootstrap technique used in the driver circuit is determined by the bootstrap capacitors $\left(C_{B 1}, C_{B 2}\right)$. As shown in Fig. 4, with large bootstrap capacitors of $1 \mathrm{pF}$, the switching time is shortened further, which is especially noticeable with a large output capacitive load. The size of the bootstrap capacitor is limited by the amount of the voltage overshoot/undershoot beyond $1 \mathrm{~V} / 0 \mathrm{~V}$ at the internal node $\mathrm{A} / \mathrm{B}$ during pullup/pulldown, which may cause the forward bias of the pn junction between source/drain-substrate. As shown in Fig. 2, with bootstrap capacitors of $0.35 \mathrm{pF}$, the voltage overshoot/undershoot at the internal node $\mathrm{A} / \mathrm{B}$ is about $0.35 \mathrm{~V} /-0.35 \mathrm{~V}$, which causes a minor leakage current of the pn junction between source/drain-substrate of the P2 and $\mathrm{P} 3 / \mathrm{N} 2$ and $\mathrm{N} 3$ devices.

Conclusion: A novel direct bootstrap technique used in a sub-l V CMOS large capacitive-load driver circuit for low-voltage CMOS VLSI has been described. For a supply voltage of $1 \mathrm{~V}$, the CMOS large capacitive load driver circuit using the direct bootstrap technique 
shows a 3.3 times improvement in switching speed in driving a capacitive load of $2 \mathrm{pF}$ compared to the conventional bootstrapped CMOS driver using an indirect bootstrap technique. This sub-1 V CMOS large load driver circuit using the direct bootstrap technique can still be advantageous at a power supply voltage of $0.8 \mathrm{~V}$.
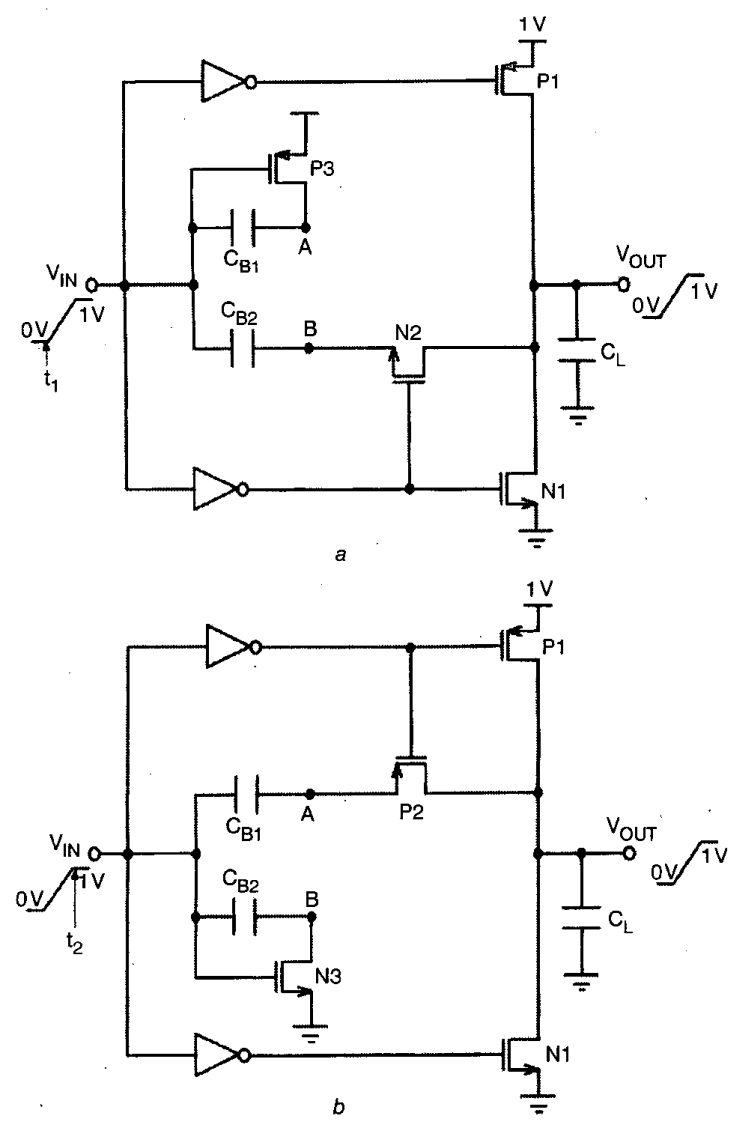

Fig. 3 Equivalent circuit of CMOS large capacitive load driver circuit with direct bootstrap technique during pullup operation

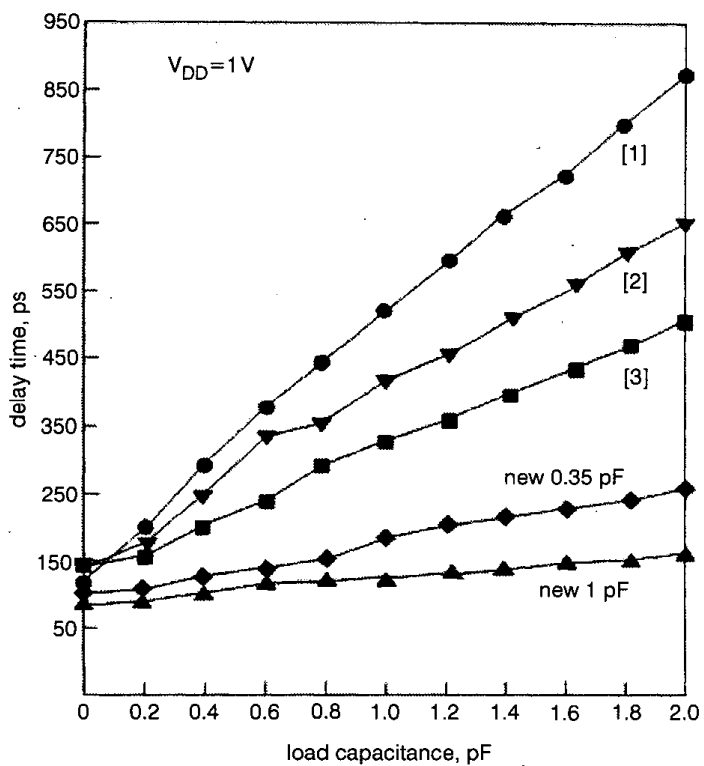

Fig. 4 Pullup delay time against load capacitance of sub-1.V CMOS large capacitive-load driver circuit with direct bootstrap technique
Acknowledgments: This work is supported under research grants from NSERC in Canada and NSC in Taiwan.

(C) IEE 2002

17 December 2001

Electronics Letters Online No: 20020208

DOI: 10.1049/el:20020208

J.B. Kuo (Department of Electrical \& Computer Engineering, DC2737, University of Waterloo, Waterloo, Ontario, Canada N2L $3 G 1)$

E-mail: jbkuo@uwaterloo.ca

P.C. Chen (Department of Electrical Engineering, National Taiwan University, Roosevelt Rd., Sec. 4, No. 1, Taipei, Taiwan 106-17)

\section{References}

l LOU, J.H., and KUO, J.B.: 'A 1.5-V full-swing bootstrapped CMOS large capacitive-load driver 'circuit suitable for low-voltage CMOS VLSI', IEEE. J. Solid-State Circuits, 1997, 32, pp. 119-121

2 LAW, C.F., YEO, K.S., and SAMIR, R.S.: 'Sub-1 V bootstrapped CMOS driver for giga-scale-integration era', Electro. Lett., 1999, 35, pp. 392-393

3 YEO, K.S., MA, J.G., and DO, M.A.: 'Ultra-low-voltage bootstrapped CMOS driver for high performance applications', Electro. Lett., 2000, 36, pp. $706-707$

\section{RNS-enabled digital signal processor design}

J. Ramírez, A. García, S. López-Buedo and A. Lloris

Residue number system (RNS) is explored for implementation of fast digital signal processors with the design of an RNS-based SIMD RISC processor. Simulations conducted on programmable logic show a sustained advantage over commercial chips for a representative set of applications, while prospective results on VLSI technology are also promising.

Introduction: Residue number system (RNS) [1, 2] arithmetic is attracting attention in the area of DSP systems owing to its high performance in Multiply and ACcumulate (MAC) intensive algorithms. However, field programmable logic (FLL) devices [2] offer the flexibility of a programmable solution and the performance of custom VLSI chips, thus enabling system integration on a single chip. In this Letter, RNS arithmetic is used to design and synthesise a high performance processor suitable for a variety of DSP applications in order to explore the possibilities of RNS for general-purpose DSP applications. Residue arithmetic performance is exploited with a single instruction multiple data (SIMD) architecture, while finite integer ring arithmetic only requires a reduced instruction set. FPL simulations were used for quantifying performance parameters such as speed and area, and for comparison with commercially available technology. Prospective results on VLSI implementations of the referred processor are also provided.

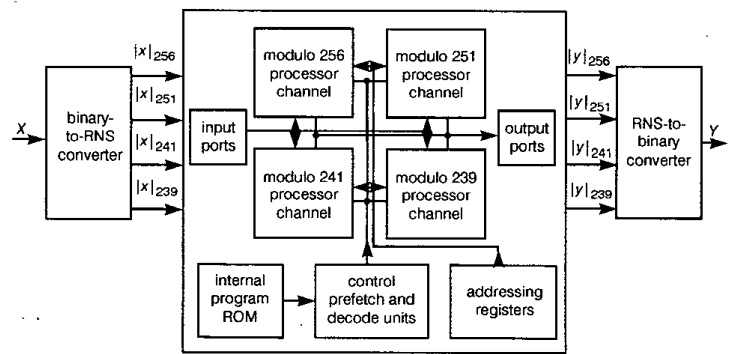

Fig. 1 Processor architecture

Processor architecture: The proposed RNS-based DSP features a RISC SIMD internal architecture with a unique program being executed over independent sets of data. Fig. 1 shows a block diagram of the processor architecture. A global input $X$ is converted to the RNS domain by a binary-to-RNS converter, residue digits $\left\{|x|_{256}\right.$, $\left.|x|_{251},|x|_{241},|x|_{239}\right\}$ are processed in parallel by the built-in 